\title{
TABLE OF LEGISLATION AND INTERNATIONAL INSTRUMENTS
}

\section{ICSID DOCUMENTS}

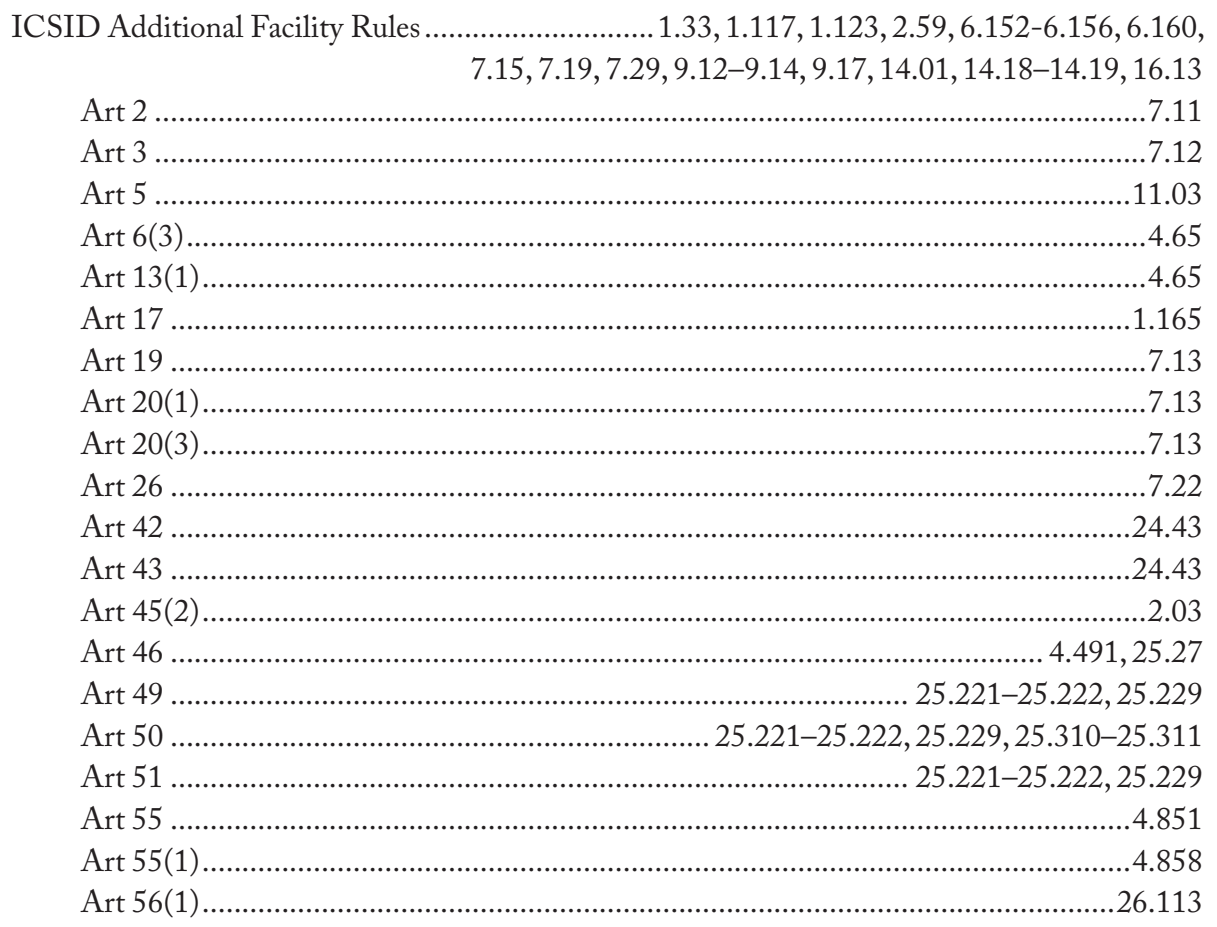

ICSID Administrative and Financial Regulations

$1.45,4.351$

Reg 1

$12.01-12.04$

Reg 1(1)

$1.46,12.01$

$\operatorname{Reg} 1(2)$

$1.48,12.02$

Reg 2 .

$1.48,12.05$

Reg 3. $1.48,12.06-12.08$

Reg 4. $1.48,12.09-12.11$

Reg 5 . $1.48,12.12-12.13$

Reg 6

$1.48,12.14$

Reg 7. $1.48,12.15-12.25$

$\operatorname{Reg} 7(3)$ 
Reg 7(4) 1.55

Reg 8 $1.80,13.01-13.04$

Reg 9 $.13 .05-13.07$

Reg 9(1) $1.82,1.86$

$\operatorname{Reg} 9(2)$ $1.82,1.86$

$\operatorname{Reg} 10$ $1.105,13.08-13.09$

Reg 11 $1.62,13.10$

Reg 12 . $.1 .62,4.04,13.11$

$\operatorname{Reg} 12(1)$ .1 .105

Reg 12(2) 1.105

Reg 13. 13.12

Reg 14 1.01-14.16, 1.106, 6.09, 16.08, 23.62

Reg 14-16

$11.03,14.01$

Reg 14(1) $4.352,6.06,14.05$

Reg 14(1) (proposed) 6.152

Reg 14(2) (proposed) 6.153

Reg 14(3)

Reg 14(3)(a) $6.43,6.45,25.223,25.291$

Reg 14(3)(d) ..4.403, 6.42-6.43, 6.46, 14.11, 14.13, 25.223, $25.229,25.291,25.312,25.321$

Reg 14(3)(e) .27 .60

Reg 14(3)(e) (proposed) 6.154

Reg 14(5) (proposed) 6.154

Reg 15. $6.43,4.17$

Reg 15(1) 20.22

Reg 16

Reg 17. $14.18-14.22,20.01,27.36$

Reg 17(2) $1.184,14.23-14.24$

Reg 17(3) 1.110

$\operatorname{Reg} 17(4)$ 1.110

Reg 17(5) 1.110

Reg 18 1.110

Reg 18(1) 1.110

Reg 18(2) 1.110

Reg 19 $1.110,14.29-14.30$

Reg 20 1.108, 15.01-15.04

Reg 21 1.108, 15.05-15.10

Reg 21(2) 1.158

Reg 22. $1.245,4.781,26.87$

Reg 22-31 .11 .03

Reg 22(1) $1.108,20.01$

Reg 22(2)

Reg 23 $1.108,24.85$

Reg 24. $1.108,16.02-16.03,16.08,20.01,26.87$

Reg 24(1) 16.04-16.06, 16.08, 20.01

Reg 24(2). 1.106

Reg 25. $.1 .106,23.44$ .1.62, 14.08, 16.07-16.09 
Reg 25(a) 1.107

Reg 25(b) .16 .09

Reg 25(c) 16.09

Reg 25(d) 16.09

Reg 26

Reg 27 $1.62,14.08,16.07-16.09,16.10-16.13$

Reg 27(1) $16.14-16.16$

Reg 27(2) .23 .26

$\operatorname{Reg} 28$ 20.22

Reg 28(1)(a) 26.82

Reg 28(1)(b) 23.36

Reg 28(2) .26 .82

Reg 29 $4.1361,27.61$

Reg 29(1) $17.01-17.05$

Reg 30 $4.802,4.1118,23.53$

Reg 30(2) 17.06-17.08, 20.01, 23.37

Reg 31 $20.18,23.37$

Reg 32 18.01-18.03, 18.04, 24.48

Reg 32(1)(a)-(b). $1.207,1.215,18.04-18.05$

$\operatorname{Reg} 32(2)(a)-(c)$ .18 .05

Reg 32(3) 18.05

Reg 33 18.05

Reg 34 19.02

Reg 34(1) 19.03-19.06, 20.03, 23.22 $11.03,20.01$

ICSID Arbitration Rules. $1.36-1.37,1.103,1.106,4.317,4.348-4.351$

Preamble $25.224,25.226$

Rule 1 $4.54,21.27,21.89$

Rule 1-6 $4.50,4.354$

Rule 1-12 .4 .969

Rule 1(1) 21.02

Rule 1(2) .4 .54

Rule 1(3)

Rule 1(4) $1.133,4.116,4.133-4.134,4.138,4.145,21.14-21.19,21.28$

Rule 2

4.1274, 21.20-21.22

Rule 2(3) 21.29

Rule 3

Rule 3(1) 21.30-21.42, 26.34

Rule 3(2)

Rule 4

Rule 4(1)

Rule 4(4) $. .4 .93,21.23-21.29,26.35$

Rule 5 $4.136-4.138,4.140,26.34$

Rule 5(1) 21.38 4.107, 21.25, 21.39, 21.43-21.62, 26.35, 27.53 $21.69,23.36$ $21.49,21.53$

Rule 5(2) 21.63-21.70, 21.89

Rule 5(3) .21 .64 $21.62,21.66$ $21.69,21.151$

Rule 6 $. .4 .55,4.926,5.04,21.71-21.86,21.89$ 
Rule 6(1)

Rule 6(2)

Rule 7

Rule 8

Rule 8-12

Rule $8(1)$

Rule 8(2)

Rule 9

Rule 9(1)

Rule 9(2)

Rule 9(3)

Rule 9(4)

Rule 9(5)

Rule 9(6)

Rule 10

Rule 10(2)

Rule 11

Rule 11(1)

Rule 11(2)

Rule 11(2)(a)

Rule 11(2)(b)

Rule 11(3).

Rule 11 (proposed)

Rule 12

Rule 13

Rule 13(1)

Rule 13(2)

Rule 13(3)

Rule 13(4)

Rule 14

Rule 14(1)

Rule 14(2)

Rule 14(3)

Rule 14(3)(e)

Rule 15

Rule 15(1)

Rule 15(2)

Rule 16

Rule 16(1)

Rule 16(2)

Rule 17

Rule 18

Rule 18(1)

Rule 19

Rule 19(2) (proposed)

Rule 19(3)....

Rule 19(4) (proposed)
21.67, 21.70, 21.73-21.74, 21.112, 23.12, 24.05 $4.926,4.1025,4.1027,21.75-21.79,21.99,21.147,27.56$

$21.70,21.73,21.87-21.91$

$5.11,5.13,21.87$

$4.1138,4.1172$

21.93-21.96, 21.142

4.111, 21.97-21.103, 21.143, 21.149

$4.973,4.1172,5.18,21.36,21.87,21.106-21.141$

$4.974,5.20,21.112,21.114-21.115$

21.119

21.119

$21.88,21.128,21.131,21.134$

$21.106,21.135$

$5.19,21.121,21.123$

$21.92,21.142-21.144,21.155$

21.143

$5.07,21.92,21.101,21.145-21.152,21.155$

$21.102,21.150-21.151$

21.148

$4.111,21.101,21.150$

21.155-21.156

21.147

6.155

21.92, 21.155-21.157

$1.106,7.20,22.02-22.24,22.42$

22.02-22.14, 22.23, 22.57, 22.60, 23.12

22.16

$22.17-22.22$

22.23

4.703, 22.15-22.30, 22.42

$22.26,22.33,22.57,22.60,23.13$

$4.353,21.143,22.27,22.58,22.60$

$22.16,22.28,22.57,22.60$

25.92

$22.15,22.25,22.31-22.36$

$22.31-22.32$

22.34

$4.695,4.702,22.37-22.46$

$21.143,22.39,22.42,22.58,23.06,26.66$

$22.42,22.57,22.60,23.48,25.54$

$22.47-22.67$

22.68-22.116, 23.19

$22.69-22.79,26.32$

$23.01-23.10,23.15,23.47,26.61$

25.96

26.22

6.156

ci 
Rule 19(5) (proposed)

.6 .158

Rule 19(6) (proposed)

6.160

Rule 20 $20.15,22.08,22.27,23.11-23.15$

Rule 20(1) $22.57,23.13$

Rule 20(1)(c) .24 .10

Rule 20(1)(d) .23 .38

Rule 20(1)(e) .24 .02

Rule 20(1)(g) 20.153

Rule 20(2)

$22.57,23.47$

Rule 21 $1.106,4.321,19.03,22.15,23.16-23.21$

Rule 21(1) $22.60,23.18$

Rule 21(2) 23.19

Rule 21 (proposed) .25 .95

Rule 22 .1.106, 23.13, 23.22-23.34

Rule 23 $1.106,23.13,23.35-23.38$

Rule 24 $23.13,23.39-23.42$

Rule 25 $23.43-23.45$

Rule 26 $20.18,23.13,23.42,23.46-23.55$

Rule 26(1)

$22.57,22.60,23.47-23.48$

Rule 26(2)

$22.57,22.60,23.48-23.49$

Rule 26(3) $23.50-23.53,24.11,25.153-25.156$

Rule 27 $4.1041,4.1127,21.114,23.56-23.61,25.153,25.157-25.158$

Rule 28 $.6 .05,6.56,23.13,23.62-23.66,26.61$

Rule 28(1) $4.353,23.63,23.66$

Rule 28(1)(a) 14.11

Rule 28(1)(b) 14.02

Rule 28(2) $23.64,23.66$

Rule 29 24.05

Rule 30

Rule 31 $22.25,23.13,24.03,24.06-24.13$

Rule 31(1) $24.06,26.50$

Rule 31(2) 24.07

Rule 31(3) $.24 .08,24.10-24.11,24.13$

Rule 32 $1.36,22.15,22.35,23.13,24.14-24.19$

Rule 32(2)

$24.16-24.18$

Rule 32(3) .24 .19

Rule 33 24.80

Rule 33(3)

Rule 34 4.317

Rule 34(1) $4.300,4.308,4.912,22.57,22.60,24.26,24.80$

Rule 34(1)(a) $2.131,2.145$

Rule 34(2) .4 .306

Rule 34(2)(a) $.4 .332,4.333,24.32-24.34$

Rule 34(2)(b) 4.1067

Rule 34(3) $4.337,24.74$

Rule 34(4) $4.317-4.318,24.38$

Rule 35 .24 .39 $4.332,22.57,22.60,24.40-24.41,24.43-24.72,24.80$

cii 
Rule 35(1) $24.55,24.60$

Rule 35(2) $24.44,24.57$

Rule 35(3) . $4.332,24.44$

Rule 35(4) 26.22

Rule 36 $24.40-24.72,24.80$

Rule 36(7) .26 .22

Rule 36(a) 24.66-24.68

Rule 36(b) 24.71

Rule 37 $1.36,1.235,4.329,4.339,4.350,24.73-24.90$

Rule 37(1) $4.337,24.73-24.74,24.79-24.86$

Rule 37(2) $24.73,24.75,24.87-24.92,24.107-24.122$

Rule 37(2)(a) $24.87,24.107-24.114$

Rule 37(2)(b) 24.115-24.119

Rule 37(2)(c) 24.110, 24.120-24.122

Rule 38 $4.324,24.131-24.149,26.21,26.36$

Rule 38(1) $4.324,26.07$

Rule 38(2)

Rule 39

$4.324,4.326,4.1065,24.134-24.137,26.07$ $1.36,2.01-25.85,4.333,4.488-4.493,4.513-4.514$, $4.523,4.632,4.639,4.651,4.658,24.04$

Rule 39(1) $4.488,4.496,4.576,25.02,25.06,25.10-25.52,25.75-25.78$

Rule 39(2) $4.488,25.02,25.53-25.55$

Rule 39(3) $4.488,4.505,25.02,25.56-25.65$

Rule 39(4) Rule 39(5)

Rule 39(6) $4.314,4.488,4.668,24.04,25.02,25.58-25.59,25.65-25.70$

Rule 40 $.25 .02,25.05,25.82-25.85$

Rule 40(1) $2.230,4.431,4.450,4.460,25.108-25.135$

Rule 40(2) $2.61,4.353,25.132,25.147$

Rule 41 $4.431,25.132,25.147$

Rule 41(1)

Rule 41(2) $2.276,4.488,25.11-25.14,25.73-25.80,25.98$

Rule 41(3)

Rule 41(4)

Rule 41(5) $2.106,4.328,4.608-4.609,6.90-6.91,22.14$, $24.04,25.55,25.176-25.191,25.195,25.313$

Rule 41(6) $2.106,4.1302,25.192-25.195$

Rule 42 $4.380,4.390,4.396,4.428,25.196-25.220$

Rule 42(1)

Rule 42(2) .4 .422

Rule 42(2)(a) .206

Rule 42(3) $4.406,4.408,4.413,23.61,25.217$ Rule 42(4)

Rule 43 $1.36,2.03,2.08,4.33,4.175,4.232-4.233,4.240,25.255$ $2.08,24.04,25.136-25.158,25.190-25.191,25.204$ $4.232,22.57,22.60,25.166-25.168$ $24.04,25.169-25.175$

Rule 43-45

Rule 43(1)

Rule 43(2)

Rule 44 $4.406,4.428,23.54,25.159-25.165,25.203-25.206$ $4.416,25.207-25.214,25.218$ $20.31,25.230$ $1.106,25.221-25.229,25.276$ 25.230-25.244, 25.247, 25.268, 25.275, 25.282 $1.106,5.16,25.243-25.266$ $4.389,4.401,4.414,4.1258,4.1264,20.31,25.267-25.285$

ciii 
Rule 44 (proposed)

$.26 .91-26.96$

Rule 45 $4.70,4.402,4.414,25.286-25.325$

Rule 45 (proposed) .26 .93

Rule 46 $4.718,23.55,26.01-26.24$

Rule 46 (proposed) .26 .95

Rule 46(1) 26.03

Rule 47 $4.695,4.712,4.938,26.25-26.69,26.104,27.61$

Rule 47(1) $22.45,26.27-26.28$

Rule 47(1)(a) .26.29-26.30

Rule 47(1)(b) 26.33-26.34

Rule 47(1)(c) 26.35

Rule 47(1)(d) $26.29,26.31$

Rule 47(1)(e) 26.36-26.37

Rule 47(1)(f) $26.38-26.39$

Rule 47(1)(g) $26.40-26.42$

Rule 47(1)(h) 26.43-26.46, 26.64

Rule 47(1)(i) $26.47-26.55,26.62,27.61$ Rule 47(1)(j) Rule 47(2) Rule 47(3) 26.65

Rule 48 $26.67-26.68$

Rule 48(1)(a) $1.36,4.718,4.784-4.785,4.801,4.938,26.70-26.96$

Rule 48(1)(b)

Rule 48(2)

Rule 48(3)

Rule 48(4)

Rule 49 $1.36,4.718,4.784-4.785,4.801,4.938,26.70-26.96$

Rule 49-52 $.6 .57,6.59-6.60,21.125,23.66,25.299,25.302,26.56-26.61,27.61$

Rule 49(1)

Rule 49(1)(c)(1) $1.112,4.801,26.76-26.80$ $4.801,26.83-26.85$ $.1 .112,4.1360,16.18$

Rule 49(1)(c)(i) $4.782,15.13,26.86-26.96$

Rule 49(1)(c)(ii)

Rule 49(2)

Rule 49(2)(c) $21.111,26.81,26.97-26.121,27.14$

Rule 49(3) $26.113,27.40$

Rule 49(4) . .4 .814$ .26 .97

Rule 49(5)

Rule 50 $26.97,26.110-26.112$ .26 .115

Rule 50(1) $22.57,22.60,26.117-26.118$ .26 .114

Rule 50(1)(c)(i) 4.917, 4.931, 4.1232, 27.08-27.44

Rule 50(1)(c)(ii) $4.852,27.10-27.11$

Rule 50(1)(c)(iii) 4.966

Rule 50(1)(d)() .27 .36

Rule 50(2) $.4 .963,27.30,27.35$

Rule 50(3) .27 .31

Rule 50(3)(a)

Rule 50(3)(b) 27.19 27.40 $4.901,4.919,4.921,27.40$
$4.963,4.1119,27.42$

civ 
Rule 50(4) 27.36

Rule 51 $4.860,4.925,27.45-27.51$

Rule 51 (proposed) 25.86-25.107

Rule 51(1) (proposed) $25.96,25.102$

Rule 51(2)(a) (proposed) 25.93-25.94

Rule 51(2)(c) (proposed) 25.98

Rule 51(2)(d) (proposed) 25.99

Rule 51(3) $4.54,4.860,27.45,27.50$

Rule 51(3) (proposed) 25.93-25.95, 25.100-25.102

Rule 51(4) (proposed) 25.102-25.104

Rule 51(5 )(proposed) 25.105-25.107

Rule 52 $27.52-27.56$

Rule 52(2)

4.1141, 27.49

Rule 52 (proposed)

25.131-25.135

Rule 53 $4.514,4.915,4.938,4.1138,4.1172,21.103,21.107,2$

$1.109,23.60,25.18,26.81,27.57-27.63,27.61$

Rule 54 4.931-4.932, 4.1174, 4.1271, 4.1288-4.1289, 4.1390, 27.64-27.81

Rule 54(1) 4.1180-4.1181, 4.1184, 4.1186, 27.65, 27.67, 27.91

Rule 54(2) $4.931,4.934,4.1184,4.1186,4.1251,27.20,27.92$

Rule 54(3) 4.1180, 4.1190, 4.1246, 4.1248, 4.1250, 4.1253, $4.1290,27.69,27.72,27.79,27.91-27.92$

Rule 54(4) $4.1200,4.1247,27.66,27.69$

Rule 54(5) 27.68

Rule 55 $1.111,4.1256,4.1258,4.1270,4.1281,27.82-27.93$

Rule 55(1) 27.92

Rule 55(2) 4.1271

Rule 55(2)(d) $4.1273,27.86$

Rule 55(3)

Rule 55(4) 21.110

Rule 56 20.33

Rule 66(1) (proposed) .25 .92

Rule10(2) 5.06

ICSID Conciliation Rules $1.36-1.37,1.103$

Rule 2(3)

Rule 9(3) (proposed)

Rule 13 $1.106,7.21$

Rule 20 20.15

Rule 21 1.106

Rule 26(2) 20.18

Rule 32 1.36

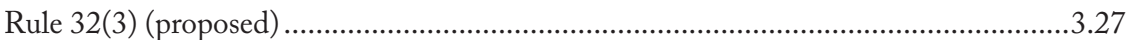

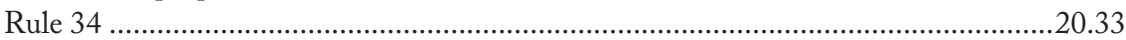

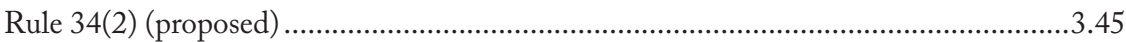

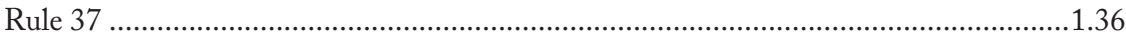

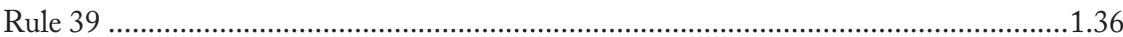

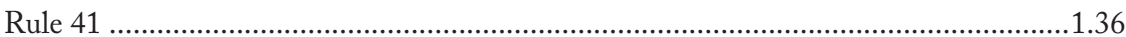


Rule 48 1.36

ICSID Convention

Preamble $.0 .01-0.95,1.192,2.34$

Recital 2 4.07

Recital 4 0.29

Recital 6 $0.28,0.37$

Recital 7

$0.28-0.29,0.40,0.42$

Art 1 1.01

Art 1(1) $1.04,2.02$

Art 1(2) 4.06

Art 2 $1.10-1.13,7.02,12.16,12.19$

Art 3 $.1 .14-1.17$

Art 4 $1.18-1.24,1.56,1.192,4.348$

Art 4(1) 9.05

Art 5 $1.25-1.31,1.72,1.192,4.33,4.348,21.52$

Art 6 $1.19,1.32-1.44,4.14,9.13,12.16$

Art 6(1)

$.1 .34,1.51$

Art 6(1)(a) $.1 .51,11.01,12.18$

Art 6(1)(b) $1.51,12.18,20.01$

Art 6(1)(c) $1.44,1.51,4.348,12.18$

Art 6(1)(d) .13 .10

Art 6(1)(f) $.1 .51,12.19,14.23$

Art 6(1)(g) 12.13

Art 7 $1.45-1.55$

Art 7(1) $1.103,1.110$

Art 7(2) .12 .17

Art 7(3) 12.05

Art 7(4) 12.18

Art 8 $1.56-157$

Art 9 $1.58-1.69,4.04$

Art 10 $1.31,1.51,1.70-1.99,2.49,4.249$

Art 10(1)

Art 10(2) $1.71,1.73,1.75,1.83,12.16,12.19,13.02$

Art 10(3)

$1.76-1.77$

Art 11 $1.81,13.05$

Art 12 $1.62-1.63,1.100-1.118,4.04,4.1360$

Art 12-16 .1.119-1.126

Art 13 $.1 .17,1.119-1.126,3.24$

Art 13(1) $1.126-1.137,15.06$

Art 13(2) $1.127,1.131,1.137,15.08$

Art 14 $1.109,1.135-1.137,15.08$

Art 14(1)

$.1 .138-1.151,5.21,21.78$ .1.138-1.141, 1.143, 1.150, 3.24, 4.155, 4.160-4.161,

4.173, 4.178, 4.180-4.181, 4.892, 4.969, 4.1074, $4.1141,10.103,21.20,21.58,21.76,21.118,27.59,59$

Art 15 $1.152-1.155,15.09$

$\operatorname{Art} 15(1)$ .1 .152

Cvi 
Art 15(3) .1 .155

Art 16 1.156-1.158

Art 16(1) 1.156

Art 16(2) 1.157

Art 16(3) $1.103,1.108,1.158$

Art 17 $1.159-1.186,10.23,14.25$

Art 18 $1.189-1.200$

Art 18-24 1.08

Art 19 1.201-1.204, 18.04

Art 20 1.190, 1.205-1.209, 18.04

Art 21 .......1.57, 1.206, 1.210-1.220, 1.224, 1.226, 1.232, 4.338, 4.558-4.559, 18.02, 24.84 Art 21(a) 1.207 Art 21(b) $1.218,1.227$

Art 22 $1.221-1.241,4.338,4.558-4.559,18.02,22.79$

Art 23 $1.242-1.249$

Art 23(1) 1.244

Art 23(2) $1.248-1.249$

Art 23(b) 1.228

Art 24 1.250

Art 24(1) .1.251-1.254

Art 24(2) $1.255-1.258$

Art 24(3) .1.259-1.261, 1.259-1.264

Art 25 $.0 .43,0.57,3.08,4.07,4.189-4.190,4.194,4.249,4.434$, $4.992,4.1166,9.10,10.24,10.69,20.07,25.128-25.129$

Art 25(1) $.0 .35,0.53,1.108,2.01-2.107,4.16-4.17,4.254$, $4.1295,10.21,10.69,15.01-15.02,20.05,25.249$

Art 25(1)(b) .10 .37

Art 25(2)(a) $2.68,2.108-2.148,4.17$ Art 25(2)(b)

Art 25(3) $2.68,2.149-2.205,4.17,4.147,24.15$ Art 25(4) $1.108,2.63-2.64,2.206-2.232,4.1300,12.21,15.01-15.02$ Art 26 $1.103,1.108,2.27,2.233-2.254,10.17,10.30,15.01-15.02$ 2.255-2.301, 2.306-2.307, 2.309, 4.18, 4.352, 4.494, 4.520, 4.554-4.557, 4.595, 4.598-4.601, 4.604, 4.1166, 4.1312,

$4.1316,4.1353,7.04,8.10,10.54,22.21,25.25,25.82$

Art 27 $2.302-2.331,4.275,4.1308,8.07,8.11,8.13,10.54,25.261$ Art 27(1) $.2 .304,4.1307-4.1311,8.10$

Art 28 $.1 .103,1.108,3.01-3.16,6.40,20.01$

Art 28(1) $3.06,20.03$

Art 28(2) $3.07,20.07$

Art 28(3) $1.111,3.08,3.13,20.24-20.25$

Art 29 $3.17,20.29$

Art 29(1) $3.18,20.14$

Art 29(2)(a) 3.19

Art 29(2)(b)

Art 30 $1.109,3.25,15.05,20.29$

Art 31 $1.123,3.17-3.27,20.29$

Art 31(1) 1.121

cvii 
Art 32 .3 .28

Art 32(1) 20.07

Art 33 $3.28,3.31,4.353$

Art 34 $3.28,3.32$

Art 34(1) .3 .33

Art 34(2) $3.35,3.38$

Art 35 $3.28,3.48-3.51$

Art 36 $1.103,1.108,1.111,2.09,4.01-4.49,4.344$ $6.40,10.54,20.01,20.03,24.03,24.48$

Art 36(1) $4.10-4.11,20.03$

Art 36(2)

Art 36(3) $3.07,4.12,4.15,20.07$

Art 37

Art 37-40 .1167, 5.06, 20.29

Art 37(1) $4.34,4.54,20.14,21.02$

Art 37(2) $3.19,4.51,4.700$

Art 37(2)(a). $4.69,21.12,26.34,27.51$

Art 37(2)(b) 4.53, 4.64, 4.72, 4.82, 4.92-4.94, 4.111, 4.116-4.117, 4.137, 4.140-4.141, 21.29-21.33, 21.42, 21.47, 26.34

Art 38 $1.109,1.192,2.179,2.205,3.25,4.33-4.34,4.50,4.55,4.70$, 4.84-4.85, 4.95-4.122, 4.168-4.169, 4.171, 4.352, 5.08, 5.23, $15.05,21.08,21.39,21.44,21.49,21.88,21.147,27.53$

Art 39 $1.133,2.179,2.205,4.51,4.123-4.153,5.23,21.14-21.15$

Art 40 1.123-1.124, 1.138, 1.151, 4.154-4.173, 4.399, 4.969, 5.23, 21.20

Art 40(1) $1.121,4.154,4.168,21.61$

Art 40(2) $.4 .51,4.155,4.172$

Art 41 $2.08,2.259,3.29,4.173-4.232,4.346,4.367,4.1157,10.54,20.27$

Art 41(1) $2.130,4.174,4.176-4.181,4.183,4.257,20.07$

Art 41(2) $4.175,4.188-4.240$

Art 41(5) 22.87

Art 42 $2.229,4.241-4.293,4.342,4.346,4.367,4.756,4.997,4.1158,22.39$

Art 42(1) $4.253-4.267,4.269,4.333,4.422$

Art 42(1)(1) 4.1074

Art 42(2) $4.255,4.268-4.269,4.704$

Art 42(3) $4.269,4.270$

Art 42(4) .4 .32

Art 43 $2.145,4.294-4.340,4.346,4.352,4.367,4.560$ $4.590,4.592,4.1159,4.1347,24.32,24.74,24.83$

Art 43(a) $4.294-4.336,4.1067,24.82$

Art 43(b) $22.15,24.74,24.81-24.82,24.84$

Art 44 $1.44,3.31,4.248,4.326,4.341-4.370,4.367,4.878,4.1160$, $4.1347,7.04,10.54,22.92,22.100,22.107,22.114,23.11,24.77$

Art 45 $.4 .346,4.367,4.371-4.429,4.1161,25.196-25.197,25.210$ Art 45(1) $4.32,23.61,25.198$

Art 45(2) $.4 .404,4.425,25.198$

Art 46 $2.230,4.346,4.352,4.430-4.480,4.1168,25.116$ 
Art 47 4.346, 4.352, 4.368, 4.481-4.683, 4.707, 4.1302, 4.1347, 25.01, $25.04-25.09,25.18,25.20,25.27,25.29,25.37,25.50,25.87,25.91-25.92$

Art 48 $4.345,4.347,4.367,4.684-4.793,4.796,4.1162,4.1347,22.39,27.61$ Art 48(1).........4.685, 4.689-4.690, 4.692-4.698, 4.707-4.708, 4.723, 21.143, 22.37, 26.66 Art 48(2) $4.685,4.711-4.718,26.65$ Art 48(3) $4.269,4.425,4.686,4.693-4.696,4.719-4.764,4.830-4.831$, 4.1078-4.1080, 4.1086, 4.1109-4.1111, 4.1115, 4.1302, 15.13, $25.114,25.237,25.264,26.45-26.46,26.48,26.53,27.61$

Art 48(4) $4.708,4.765-4.777,26.03,26.67$ Art 48(5) $4.778-4.793,15.12,26.86,26.92$ Art 49 $4.347,4.367,4.794-4.849,4.1353,6.40,16.17,24.131,25.44,25.265,27.63$ Art 49(1)................. 1.103, 1.112, 4.794-4.802, 4.798-4.802, 4.1360, 26.03, 26.83-26.84 Art 49(2)...... $1.111,4.737-4.740,4.744,4.794-4.797,4.803-4.811,4.820,4.827$, 4.831-4.844, 4.923, 4.939, 4.1111, 4.1118, 4.1347, 10.54, $21.111,25.226,25.238,25.278,26.84,26.97$ Art 50 .1.111, 4.345, 4.347, 4.808, 4.850-4.860, 4.939, 4.1170, $4.1353,6.40,10.54,25.226,25.238,25.265,27.02$

Art 50(1) $1.103,4.852,27.12$

Art 50(2) $4.487,4.514,4.860,4.1174,27.45,27.64$ Art 50(3)(a) $26.84,27.40$ Art 50(3)(b) Art 51 26.84 $4.324,4.345,4.347,4.804,4.861-4.939,4.974,4.1122$, 4.1146, 4.1170, 4.1353, 6.40, 10.54, 24.139-24.142, $25.226,25.238,25.265,27.02,27.15$

Art 51(1) $1.103,4.898-4.914,24.139-24.140$ Art 51(2) $4.794,4.847,4.884,4.887,4.915-4.923,4.11122$

Art 51(3) 4.924-4.927, 4.1174, 27.45

Art 51(4) 4.487, 4.928-4.930, 4.928-4.940, 4.1174, 27.64, 27.70

Art 51(a) 4.906

Art 52 $1.111,1.210,2.06,4.241,4.347,4.367,4.736,4.741,4.752$, 4.831, 4.939, 4.1088, 4.1111, 4.1170, 4.1232, 4.1353, 6.40, $10.54,25.226,25.238,25.265,26.33,26.62,27.02,27.61$

Art 52(1) $1.103,1.141,2.06,4.309,4.940-4.966,4.1168,4.1170,4.1773,27.27$ Art 52(1)(a) $4.105,4.720-4.721,4.891,4.966-4.985,4.967-4.985$,

4.1023, 4.1027, 4.1077, 4.1314, 21.114, 22.83, 26.33

Art 52(1)(b) $4.41,4.243,4.742,4.889,4.966,4.986-4.1018,4.1034,4.1040,22.90$ Art 52(1)(c) 4.887, 4.966, 4.1019-4.1032, 4.1030, 4.1037 Art 52(1)(d) 4.343, 4.697, 4.742, 4.889, 4.966-4.967, 4.975, 4.980, 4.1026, 4.1033-4.1077, 26.13, 26.16, 26.39, 26.117 Art 52(1)(e) 4.697, 4.744, 4.758, 4.831, 4.889, 4.966, 4.1034, $4.1078-4.1117,25.114,25.265,26.48$

Art 52(2) 4.794, 4.847, 4.887, 4.1028, 4.1033, $4.1118-4.1129,4.1183,4.1217,4.1272,25.278$ Art 52(3) $1.109,1.121,1.134,2.179,2.205,4.124,4.558,4.947$, 4.960-4.961, 4.1130-4.1155, 14.02, 15.05, 27.54 Art 52(4).4.368, 4.398, 4.514, 4.795, 4.820, 4.885, 4.1156-4.1172, 4.1164, 4.1167, 4.1172, $6.132,7.07,14.01,22.107,25.18,25.20,25.92,26.81,26.100,27.59,27.61$ 
Art 52(5)

Art 52(6)

Art 53

Art 53(1)

Art 53(2)

Art 54

Art 54(1)

Art 54(2)

Art 54(3)

Art 55

Art 56

Art 56-58

Art 56(1)

Art 56(2)

Art 56(3)

Art 57

Art 58

Art 59

Art 60

Art 60(1)

Art 60(2)

Art 61

Art 61(1)

Art 61(2)

Art 62

Art 63

Art 63(a)

Art 63(b)

Art 64

Art 65

Art 66

Art 66(1)

Art 66(2)

Art 67

Art 68

Art 68(1)

Art 68(2)

Art 69

Art 70
..4.368, 4.487, 4.514, 4.849, 4.1169, 4.1173-4.1255 $4.1271,4.1289,27.64,27.70,27.75$ $4.345,4.1122,4.1256-4.1290,6.03,21.110,27.86$ 4.367, 4.572-4.575, 4.1203, 4.1229, 4.1234 4.1291-4.1334, 7.04, 8.10-8.11, 8.14, 10.54, 25.240 $2.06,4.850,4.940,4.1174,4.1202,4.1233,4.1294,4.1298-4.1309$, $4.1317,4.1324-4.1325,4.1329,4.1347,4.1366 .4 .1353,26.28$ 4.939, 4.1301, 4.1347, 27.63 $2.06,3.43,4.367,4.685,4.1203-4.1204,4.1240,4.1244$ $4.1320-4.1331,4.1335-4.1367,7.04,8.10,10.21,10.23,25.240$ $1.103,4.04,4.275,4.1310,4.1322,4.1324-4.1327$, 4.1343-4.1359, 4.1366, 26.37 $1.108,4.1348,4.1360-4.1364,10.21,15.01-15.02$ $4.1360,4.1365-4.1366$ 2.06, 4.1229, 4.1231, 4.1234, 4.1320, 4.1345, 4.1353, $4.1367-4.1399,4.1383,8.10,10.18,25.240$ $4.347,4.797,5.01-5.02,21.87,21.107$ $5.01,21.107$ $.5 .02,5.04,5.06-5.07,5.10,22.99$ .5 .05 $.1 .109,1.121,5.02,5.10-5.17,21.100$ 4.151, 4.347, 4.1008, 4.1025, 4.1077, 5.01, 5.03, 5.18-5.59, 21.36, 21.76, 21.87, 21.106-21.107, 21.124, 21.129, 21.133, 22.113, 41075 $.1 .109,4.347,5.01,5.60-5.64,21.79,21.87,21.106-21.107,21.128$ $1.103,1.106,6.02 .6 .07,6.17-6.20,6.38-6.44,6.132,14.02,20.20$ $1.106,1.159,4.347,4.352,6.02,6.07,6.17,6.24-6.27,6.46-6.50,6.132,14.02$ $1.103,6.21-6.22,6.46-6.48$ $6.23,6.49-6.50$ $1.159,4.347,4.352,6.02,6.17,6.28-6.37,6.51-6.65,6.132,6.161,14.02$ $3.35,6.05,6.51-6.54$ $4.1163,6.05,6.23,6.55-6.65,21.125,23.64,23.66,24.39$, $25.239,25.262,25.293-25.295,25.299-25.301,25.303,26.56$ $4.352,7.01-7.23,7.28,7.43,16.11$ $4.352,7.01,7.07-7.08,7.20,7.22,7.24-7.50,16.11,22.17-22.20$ .7.24-7.26 $1.103,7.27-7.29$ $4.211,4.1311,8.01-8.26,10.92$ $1.103,1.110,4.1316,9.02-9.06,9.10-9.11,9.13,9.17,9.39,10.03$ 4.1316, 9.02-9.03, 9.07-9.11, 9.13, 9.17, 9.39, 10.03, 10.30, 10.94, 21.130 $.1 .110,9.09-9.10,12.19$ ... 9.10 $1.20,1.108,1.192,9.22,10.01-10.09,10.28,10.94,12.19$ $1.108,10.10,10.30,10.94,10.98$ $4.1335,10.10$ $10.10,10.14$ $.1 .108,4.1344,10.18-10.24,15.01-15.02$ $1.108,1.201,2.178,10.17,10.25-10.32,10.94,15.01-15.02$ 
Art 71 $.1 .108,2.79,2.84,4.1335,10.16,10.33-10.81,10.37,10.94,10.99$

Art 72 $2.84,10.16,10.31,10.33-10.81$

Art 73 $1.108,10.30,10.82-10.88$

Art 74 $10.89-10.93$

Art 75 $10.30,10.94-10.99$

Art 75(a) 10.08, 10.94-10.99

Art 75(b) $10.11,10.94-10.99$

Art 75(c) $10.94-10.99$

Art 75(d) 10.31, 10.94-10.99

Art 75(e) 10.94-10.99

Art 75(f) 10.94-10.99

Final clause $10.100-10.104$

ICSID Convention [Preliminary Draft October 1963][Doc 24]

0.51, 1.05, 4.804, 4.987 Preamble. 0.29

Art I, Sec 1 - Sec 3 1.26

Art I, Sec 9(2) $1.77-1.78$

Art I, Sec 10(2) 1.81

Art I, Sec 16 $1.162-1.164$

Art 1, Sec 18(2) 1.229

Art II, Sec 3 $4.179,4.215$

Art II, Sec 3(1) 4.180

Art II, Sec 3(2) $4.189,4.215$

Art II, Sec 3(3)

Art II, Sec 16

$2.182,2.185$

Art IV, Sec 2(2)

1.162-1.164

Art IV, Sec 6

4.126-4.127

Art IV, Sec 7(2) .4 .779

Art IV, Sec 10 4.798-4.799

Art IV, Sec 11(1) .25 .28

Art IV, Sec 12(2)

$4.799,4.858$

Art IV, Sec 13 4.799

Art IV, Sec 13(4) $.4 .752,4.1022,4.1037$ $4.799,4.968$

Art IV, Sec 14 4.1293-4.1294, 4.1297

Art VI, Sec 1 $.6 .13,6.22,6.28$

Art X, Sec 2 ..1.77

Art XI, Sec 1 10.05

Art XI, Sec 2 10.19

Art XI, Sec 5 10.38

ICSID Convention [Working Paper 1962][Doc 6]

Art II ... 4.1297

Art III, Sec 1

Art III, Sec 9

Art III, Sec 11(2)

$1.77-1.78$

Art III, Sec 12(2)

Art III, Sec 20(2) 
Art IV, Sec 3 4.215

Art IV, Sec 4 $2.150,2.182$

Art VI .1 .32

Art VI, Sec 6 4.858

Art VI, Sec 1

.1 .34

Art VI, Sec 10

$4.1294,4.1297$

Art VII, Sec 1

$6.12,6.18,6.21,6.28$

Art VII, Sec 5

Art X, Sec 2 .

ICSID Convention [First Draft September 1964][Doc 43] Art 17

$1.164-1.166$

Art 25

10.19-10.20

Art 29

$2.237-2.238$

Art 30

$2.156,2.185,2.191,2.205$

Art 43(1)

4.128

Art 44(1)

4.220

Art 48(2)

4.717

Art 49

.4 .434

Art 50

.25 .28

Art 51

$4.796,4.800,4.806-4.807$

Art 51(1)

$.4 .752,4.779,4.808$

Art 51(2)

4.808

Art 52 4.800-4.801, 4.804

Art 52(3) 4.808-4.810

Art 53 $4.808,4.1294$

Art 54 $4.804,4.807$

Art 55(1).

$4.968,4.1037$

Art 55(1)(b)

.4 .805

Art 55(1)(d)

4.805

Art 56

4.1294, 4.1297

Art 62

$6.28,6.33$

Art 63

.6 .14

Art 64(1)

6.22

Art 64(2)

6.23

ICSID Convention [Revised Draft December 1964][Doc 123]

Art 17

.1.165-1.166

Art 23 1.244

Art 28 2.156

Art 39

4.130

Art 40(1)

4.130

Art 49(1)

$4.796,4.801$

Art 49(2)

$4.796,4.810$

Art 52(1)

Art 53

Art 53(1)

4.1297

Art 53(2) 
Art 59 6.19

Art 60(2) 6.23

Art 61(1). 6.35

Art 69 10.20

ICSID Institution Rules 4.352

Rule 1 $.1 .111,19.03,20.03-20.04$

Rule 1(1) .23 .22

Rule 1(2) 20.30

Rule 2 4.18, 20.01, 20.05-20.12

Rule 2(1) 2.225

Rule 2(1)(a) $22.73,26.29$

Rule 2(1)(b) 4.09

Rule 2(1)(c) $4.09,20.07$

Rule 2(1)(d) $2.179,2.204,4.147-4.148,20.07$

Rule 2(1)(e) 20.11

Rule 2(1)(f) 20.07

Rule 2(2) $2.225,20.07,20.25$

Rule 3 20.13-20.15, 23.05

Rule 4 17.06, 20.16-20.19

Rule 5 20.01, 20.20-20.23, 20.32

Rule 5-8 1.111

Rule 5(2) 20.16

Rule 6 $4.25,4.37,20.24-20.28$

Rule 6(1)(a) 25.10

Rule 6(1)(b) 2.117

Rule 6(2) $2.09,25.10$

Rule 7 20.01, 20.29

Rule 7(e) $2.09,20.27$

Rule 8 $20.20,20.30-20.32,25.225$

Rule 9 20.33

Rule 9(2) 20.01

ICSID Memorandum of Fees and Expense 2005 6.47

ICSID Model Clauses for Use in Bilateral Investment Agreements 1969

$2.322,4.355$

$\mathrm{Cl} 52.223$

$\mathrm{Cl} 12$ .2 .270

$\mathrm{Cl} 17$ 4.355

ICSID Practice Notes

Practice Notes for Respondents in ICSID Arbitration $4.12,4.27$

ICSID Rules and Regulations 1968 $9.12-9.16,11.01$

Rule 8

Rule 9 $21.114,24.146$

Rule 9(1) .24 .146

Rule 11 $21.145,21.152$

cxiii 
Rule 13 22.31

Rule 17 $22.47-22.49$

Rule 27 .24 .145

Rule 32 9.15

Rule 35 .24 .41

Rule 37 .9 .15

Rule 41(5) .20 .28

Rule 43 .25 .234

Rule 44 .25 .294

Rule 45 .25 .287

Rule 46 $26.02-26.05$

Rule 47 $26.25,26.65-26.66$

Rule 48 $9.15,26.71-26.74$

Rule 49

21.111

ICSID Schedule of Fees 2017 $6.47,6.49-6.50$ para 1 .6 .40

para 3 6.49

\section{INTERNATIONAL ORGANISATION DOCUMENTS}

African Development Bank

Charter

$$
\text { Art } 50
$$

Court of Justice of the European Union

Rules

Art 44 .4 .864

International Bank for Reconstruction and Development

Articles of Agreement.

$1.252-1.253$

Art VII. $1.190,10.20$

Memorandum of Administrative Arrangements between the IBRD and ICSID, 13

February 1967 (Doc. AC/66/6)

Memorandum on Signature and Ratification, Acceptance or Approval of the ICSID Convention, 1965

International Court of Justice

Rules 1946

Art 38(1) .4 .266

Art 38(1)(a) 4.362

Art 46(5) 4.218

Art 61(8) 25.67

Rules 1978

Art 2 
Art 9 .1 .148

Art 36 4.375

Art 36(1) 8.01

Art 37 4.375

Art 38 8.26

Art 38(1)(d) 4.731

Art 40(1) 8.26

Art 41 $4.486,25.09$

Art 41(1) 4.486

Art 42 .22 .69

Art 42(1) 22.72

Art 42(2) .22 .72

Art 53 $4.375,4.386$

Art 55 .4 .701

Art 57 4.765

Art 58 4.715

Art 60 4.851

Art 61 $4.864,4.901,4.907,4.909$

Art 79 4.218

Art 80 4.361

Art 80(1) 4.434

International Criminal Court

Rules

Art 6(3) 4.375

Art 6(4)

Art 29

Statute of Rome 1998

Preamble

International Development Association

Articles of Agreement 24 September 1960,

Article XI, Sec 3

International Law Commission

Draft Articles on Diplomatic Protection 2006

Draft Articles on State Responsibility for Internationally Wrongful Acts 2001

$2.229,4.679$

Art 3

Art 4

Art 8

Art 13

Art 25 4.1001, 4.1089

Art 35 4.562

Model Rules on Arbitral Procedure

Art 11

Art 28(1) 
Art 35(b)

$4.1020,4.1022$

Art 35(c)

Art 36(2)

International Monetary Fund

Articles of Agreement

Art 2, s II

International Tribunal for the Law of the Sea

Statute

Art 28 4.375

Art 30(1)

Art 30(3)

Art 30(4)

4.715

NAFTA Free Trade Commission

Joint Statement, Decade of Achievement (16 July 2004)

United Nations

Charter

Art 92

Art 102

Art 102(1)

$10.90-10.91$

World Bank

Articles of Agreement. .10 .05

Art 2 9.22

Article VII, section 9(c) .1 .263

\section{NATIONAL LAWS}

Benin

Decree No. 445/PR/MFAEP of 28 December 1967 regarding nomination on the list of

ICSID conciliators and arbitrators (Off. Gaz. 4, 14 February 1968, 161)

Cameroon

Law 75-18 of 8 December 1975 regarding recognition of arbitral awards (Off. Gaz. 6, Suppl., 15 December 1975, 234) $10.21,10.23$

Denmark

Act No. 466 of 15 December 1967, on Recognition and Execution of Orders Concerning Certain International Investment Disputes $10.21,10.23$

Finland

Decree No. 75/69 of 24 January 1969, containing regulations for the implementation of the Convention .10 .23

France

Code of Civil Procedure 2011 
Art 1465 4.177

Art 1484(1) 4.988

Art 1484(2)(1) 4.988

Art 1484(2)(2)

Art 1484(2)(3) 4.988

Constitution

Art 55

Preamble 0.11

Germany

Zivilprozessordnung 2005

Section 1040(1)

Ireland

Arbitration Act 1980

s 1510.23

International Centre for Settlement of Investment Disputes (Designation and Immunities) Order, 1980 (SI No. 339 of 1980)

Jamaica

Investment Disputes Awards (Enforcement) Act, 1966 (Appointed Day) Notice. (Notice No. 45 of 7 February 1967) (Off. Gaz.XC, 18, 16 February 1967, 60) ...... 10.21, 10.23

Malawi

Investment Disputes (Enforcement of Awards) Act, 1966 (Act 46 of 29 December 1966)

(Off. Gaz. Suppl., 10 January 1967) $10.21,120.23$

Mauritius

Decree No. 49 of 1967, International Centre for Settlement of Investment

Disputes (Enforcement of Awards) (Off. Gaz. Extr. 105, Vol. 54, No. 30, 1967, p. A255)

Investment Disputes (Enforcement of Awards) Act, 1969 (Act No. 12 of 24 April 1969) ... 10.23

New Zealand

Arbitration (International Investment Disputes) Act 1979

Arbitration (International Investment Disputes) Amendment Act 2000.

Sweden

Act on Recognition and Execution of Awards Concerning Certain International Investment Disputes (Act No. 735 of 16 December 1966).

Arbitration Act 1999

Art 34(1)(1) 4.988

Art 34(1)(2)

Art 34(1)(4)

Art 34(1)(5) 4.969 
Switzerland

Federal Private International Law Statute 1987

Art 190(1)2(a)

4.969

Art 190(1)2(b)

4.988

Art 190(1)2(c)

4.988

Trinidad \& Tobago

Investment Disputes Awards (Enforcement) Act, 1968 (Act No23 of 13 August 1968)...... 10.23

United Kingdom

Arbitration (International Investment Disputes) Act 1966

$$
\text { s } 14.1349
$$

Arbitration Act 1996

$$
\begin{aligned}
& \text { s } 304.177 \\
& \text { s } 674.969,4.989 \\
& \text { s 68(2)(b) } \\
& \text { s 68(2)(d) }
\end{aligned}
$$

State Immunities Act 1978

\section{United States}

Civil Code, 19 USC

$$
\text { s } 2462(\mathrm{~b})(2)(\mathrm{E})
$$

Convention on the Settlement of Investment Disputes Act 1966, 22 USC s 1650

Delaware General Corporation Law

$$
\text { s } 278 \text {. }
$$

Executive Order designating certain Public International Organizations entitled to enjoy certain privileges, exemptions and immunities (Exec. Order 11966; 42 Fed. Reg. 4331 (1977)).

Federal Arbitration Act 1925, 9 USC $4.1358,7.31$ s10(a)(2) 4.969 s10(a)(4)

Foreign Relations Code, 22 USC

$$
\text { s } 1650 \mathrm{a} \text {. }
$$

Foreign Sovereign Immunities Act, 28 USC

s 1391(f)

s 1608

$$
\text { s } 1610(a)(1)
$$

Venezuela

Investment Law

Art 22 $.0 .41-0.43$ 


\section{OTHER ARBITRATION RULES}

American Arbitration Association Rules 2013

Art 28 25.218

Art 32.1 25.246

Art R-38 4.497

American Chamber of Commerce Arbitration Rules 2014 (AMCHAM)

Art 13.6

Australian Centre for International Commercial Arbitration Expedited Arbitration Rules 2016 (ACICA)

Art 25

Chartered Institute of Arbitration

International Arbitration Guideline on Interviews for Prospective Arbitrators

Guidelines 7-8.

China International Economic and Trade Arbitration Commission Arbitration Rules 2015

(CIETAC)

App III 4.497

Art 30

Art 45(2) 26.01, 26.06

Art 45(3) 26.11

Art 47(1) $26.27,26.83$

Art 47(2) 26.67

Art 47(3).

Art 48 $26.12,26.25$

Art 48(1) .26 .06

Art 48(2). 26.11

Art 50 $26.99,26.110$

Art 50(2) 26.84

Art 51 $26.99,26.104$

Art 53(3). 26.57

Commercial Mediation and Arbitration Commission of the Mexico City National Chamber of Commerce Arbitration Rules 2008 (CANACO Rules)

Art 33 ... 25.217

Art 33(1) 25.218

Art 33.1 25.219

Hong Kong International Arbitration Centre Rules 2013 (HKIAC)

Art 7 25.217

Art 26 $25.217,25.218$

Art 26.1 25.219

Art 34.5 .26 .01 
Sch 4

Institut de Droit International

Draft Rules for International Arbitration Procedure, 1875

Art 27

International Bar Association

Guidelines on Conflicts of Interest in International Arbitration.

Guidelines on Party Representation in International Arbitration

Rules of Ethics for International Arbitration

Art 3.1

1.146

Rules on Taking of Evidence in International Arbitration.

$2.145,4.312-4.313,22.11$

Art 3.10

Art 4.3

.24 .46

Art 4.5

24.67

Art 4.8

24.69

Art 5.2

.24 .63

Art 5.2(a)

.24 .52

Art 5.2(c)

.24 .52

Art 8.2 $24.58,24.60,24.62$

Art 8.3(a)-(c)

24.55

Art 8.3(f)

24.64

Art 8.4

24.56-24.57

Art 9.1 4.312

Art 9.2(e) .24 .60

Art 9.2(f) 24.60

Art 9.2(g) 24.60

Art 24 .24 .46

International Centre for Dispute Resolution Procedures 2014 (ICDR Rules)

Art 26

Art 30(1) $26.01,26.06$

International Chamber of Commerce Arbitration Rules 2017 (ICC Rules)

Appendix 3, Art 2

Art 6(4)

Art 6(5)

Art 6(8)

Art 11(1)

Art 13(5)

Art 23(1)(c)

Art 23(1)(d)

Art 25

Art 26(2)

Art 31

Art 31(1) $26.01,26.06$

Art 31(2) 26.11 
Art 32(1) .4 .706

Art 33 $25.246,25.249$

Art 34 26.75

International Institute for Conflict Prevention and Resolution Global Rules for Accelerated Commercial Arbitration 2009 (CPR Rules)

Rule 14 $25.216-25.2187$

Japan Commercial Arbitration Association Rules 2015 (JCAA Rules)

Rule 71

Rule 72

Kigali International Arbitration Centre Arbitration Rules 2012 (KIAC Rules)

Art 7

Kuala Lumpur Regional Centre for Arbitration, Arbitration Rules 2017 (KLRCA Rules)

Art 30

Art 30(1)(a)

London Court of International Arbitration (LCIA)

Arbitration Rules 2014

Art 15.8

Art 20

Art 22

Art 26.2

Art 26.9

Schedule of Arbitration Costs 2014 para 2(i).

6.48Permanent Court of Arbitration Arbitration Rules 2012 (PCA Rules) .4.433

Art 23(1)

Art 34(2) 26.28

Art 34(3)

.26 .27

Art 34(4) $26.01,26.65,26.83$

Art 38 26.99

Art 38(2) 26.84

Art 39 $26.99,26.104$

Art 42(1) $6.61,26.57$

Singapore International Arbitration Centre Investment Arbitration Rules 2017 (SIAC Rules)

Rule 2 26.84

Rule 4.1(b) 25.135

Rule 17 25.135

Rule 17.8 25.217

Rule 17.9 25.217

Rule 30.3 $.26 .01,26.06,26.75$

Rule 30.4 26.27

Rule 30.11 26.28 
Rule 31 .26 .110

Rule 31.1 $26.84,26.99$

Rule 31.2 . 26.99

Rule 31.3. $26.99,26.104$

Rule 35 26.57

Sch 1 4.497

Stockholm Chamber of Commerce Arbitration Rules 2017 (SCC Rules)

App II 4.497

Art 5 .26 .84

Art 13(5) .21 .14

Art 32 .24 .43

Art 33 .24 .43

Art 33(1) 25.218

Art 35(1) 25.218-25.219

Art 35(2) 25.217

Art 35(3) 25.218

Art 42(1) 26.27

Art 42(2) .26 .83

Art 42(3) .26 .65

Art 43 $26.01,26.06,26.11$

Art 46 26.28

Art 47 $26.99,26.110$

Art 47(1) .26 .84

Art 48 $26.99,26.104$

Art 50 26.57

Swiss Chamber Arbitration Institute Rules 2012 (SCAI Rules)

Art 34.1 .25 .246

Swiss Rules of International Arbitration Rules 2012 (SRIA Rules)

Art 43 4.497

UNCITRAL Arbitration Model Law on International Commercial Arbitration 2006

Art 11(5) 21.14

Art 16(1)

Art 17A

.4 .655

Art 20(2)

.7 .18

Art 30

$25.246,25.249$

Art 34

Art 34(2)(a)(ii) $4.969,4.1035$

Art 34(2)(a)(iii) 4.988

Art 34(2)(a)(iv) 4.969, 4.1035

Art 34(2)(b)(ii),

Art 36

Art 36(1)(a)(ii) $4.969,4.1035$

Art 36(1)(a)(iii) .4 .988 
Art 36(1)(a)(iv)

$4.969,4.1035$

Art 36(1)(b)(ii).

4.1020

UNCITRAL Arbitration Rules 1998.

$.2 .148,4.122,4.434,4.789,7.15$

Art 2

.26 .84

Art 2(2)

26.77

Art 3(1)

20.04

Art 6(7)

21.14

Art 11(1)

21.116-21.117

Art 12(1)

$1.145,5.29$

Art 15

.24 .76

Art 15(1)

$24.78,24.95$

Art 16

Art 18

4.1049

Art 21(3)

4.452

Art 24

4.299

Art 25

4.299

Art 27

$4.299,24.43$

Art 27(1)

.4 .303

Art 28

24.43

Art 30

25.217-25.218

Art 30(1)(a)

25.218-25.219

Art 31(1)

.4 .715

Art 33(2)

4.706

Art 34(2)

26.28

Art 34(3)

$26.27,26.65$

Art 34(4)

26.01, 26.83

Art 37

.4 .858

Art 38

$26.99,26.110$

Art 38(1)

26.84

Art 39

$26.99,26.104$

Art 40(1)

6.61Art 41

6.48Art 42

6.61Art 42(1)

UNCITRAL Notes on Organizing Arbitral Proceedings 1996

7.15-7.18, 7.30, 7.32, 7.46, 7.50, 24.55

UNCITRAL Rules on Transparency in Treaty-based Investor-State Arbitration 2014

4.788-4.789, 26.87, 26.89

Art 1.1

24.100

Art 1(9)

24.104

Art 3.1

$4.788,4.790$

Art 3.2

4.790

Art 4(1)

24.103

$\operatorname{Art~4(2)(c).~}$

24.103

cxxiii 
Art 4(4)(d) .24 .101

Art 5(2)

Art 5(5)

Art 7

Vienna International Arbitral Centre Rules

Art 36(2) 26.01

World Intellectual Property Organization Arbitration and Mediation Center Arbitration Rules 2014 (WIPO Rules)

Art 58

Art 58(a)

World Trade Organization Dispute Settlement Understanding

Art 14:3

Art 17:11

\section{OTHER CONVENTIONS AND AGREEMENTS}

Agreement among the Government of Japan, the Government of the Republic of Korea and the Government of the People's Republic of China for the Promotion, Facilitation and Protection of Investment 2012

Art 1(2) 2.69

Art 1(4)

Agreement on Promotion, Protection and Guarantee of Investments amongst the Member

States of the Organization of the Islamic Conference ('OIC Investment Agreement').2.77

Albania-Greece Bilateral Investment Treaty 1991

Algeria-Italy Bilateral Investment Treaty 1991

Art 1(1)

Argentina-France Bilateral Investment Treaty 1991

Argentina-Germany Bilateral Investment Treaty 1991

Argentina-Peru Bilateral Investment Treaty 1994

Art 1(1)

Argentina-Spain Bilateral Investment Treaty 1991

Art II (2)

Argentina-Spain Bilateral Investment Treaty [date]

Art IV(2)

$2.105,4.469$

Art X.

$\operatorname{Art} \mathrm{X}(3)(\mathrm{a})$

Argentina-UK Bilateral Investment Treaty 1990

Argentina-US Bilateral Investment Treaty 1991

$\operatorname{Art~II(2)(c)~}$ $4.1092-4.1093$

Australia-Argentina Bilateral Investment Treaty 1995

Australia-Chile Free Trade Agreement [date]

Art 10.16.2

Art 10.16.4 
Art 10.20 .26 .89

Art 10.21.2 26.89

Art 10.22(1) 26.89

Art 10.26 26.89

Australia-Indonesia Bilateral Investment Treaty 1992

Austria-Croatia Bilateral Investment Treaty 1997

Art 11(1)

Austria-Czech Republic Bilateral Investment Treaty 1993

Art 11

Bangladesh-Italy Bilateral Investment Treaty 1990

Art 1(1)(c).

Belgium and Luzembourg-Burundi Bilateral Investment Treaty 1989

Art 8

Belgium-Russian Federation Bilateral Investment Treaty 1989

2.104

Belgium-Luxembourg-European Union-China Bilateral Investment Treaty 1986

Bolivia-Chile Bilateral Investment Treaty 1994

Art II

Bolivia-Chile Bilateral Investment Treaty [date]

$\operatorname{Art~I}(2)$

Art X

Bolivia-UK Bilateral Investment Treaty 1988.

Brussels Convention 1963 Supplementary to Paris Convention on Nuclear Energy 1960

Art 5(a)

Canada-Costa Rica Bilateral Investment Treaty 1998

Art $1(\mathrm{~g})$

Canada-EU CETA 2016

Art 8.23

Art 8.27(4)

Art $8.28(2)$ $4.1314-4.1315$

Art 8.28(4)

Art 8.29

Art 8.30

Art 8.39(5)

Canada Model Bilateral Investment Treaty 2004.

Canada-Mongolia Bilateral Investment Treaty 2016

Art 25(1)...

Art 25(2)

Art 25(4)

Art 27(1)

Canada-Peru Bilateral Investment Treaty 2006

Canada-Peru Free Trade Agreement 2009

Art 836

Canada-Peru Free Trade Agreement 2008

Annex 836.1.

Canada-Slovakia Bilateral Investment Treaty 2010

Art 15(6)

Canada-Venezuela Bilateral Investment Treaty 1996 
Art 1(f)

Art $1(\mathrm{~g})$

Chile-Peru Bilateral Investment Treaty 2000

Chile-Spain Bilateral Investment Treaty 1991

Art 10(2)

Chile-Venezuela Bilateral Investment Treaty 1996

China-Germany Bilateral Investment Treaty 2003

Art 1

Art 9

Protocol

China-Hong Kong Bilateral Investment Treaty 2016

Art 26(5)

Art 32(3)

China-Korea Bilateral Investment Treaty 2007

Art 9(7)

China-Netherlands Bilateral Investment Treaty 2001

Art 10(4)

Colombia-France Bilateral Investment Treaty 2014

Art 15(18)

Comprehensive and Progressive Agreement for Trans-Pacific Partnership 2018

Art 9.19.2

Art 9.22(2)

Art 9.22(5)

Art 9.22(6)

Comprehensive Economic and Trade Agreement

Art 8.27(17)

Convention establishing the Multilateral Investment Guarantee Agency 1985

Art 66 .10 .26

Convention on Discrimination against Women 1979

Art 29

Convention on International Settlement Agreements Resulting from Mediation 2018

(Singapore Convention).

Convention on the International Telecommunication Union 1992

Convention on the Privileges and Special Immunities of the Specialised Agencies 1947 ....1.202

Council of Europe Convention against Trafficking in Human Organs 2015

Art 4

Croatia-Azerbaijan Bilateral Investment Treaty 2007

Art 13(5)

Czech Republic-Israel Bilateral Investment Treaty 1996

$2.42,2.44$

Dominican Republic-Central America Free Trade Agreement 2016 (CAFTA-DR)2.77, 24.101

Art 10.1.3

.2 .103

Art 10.12.2

2.318

Art 10.20(2)-(3).

Art 10.20.5

Art 10.21

Ch. 10, Ann. 10-F(1)

4.1314

Economic Cooperation Agreements 
Netherlands-Indonesia 1968

Art 11 4.247

Egypt-Cyprus Bilateral Investment Treaty 1998 .....

El Salvador-Spain Bilateral Investment Treaty 1995

$2.44,2.51$

Energy Charter Treaty 1994

$2.42,2.48,2.51,2.77,2.88,24.130$

Art 1(6)

Art 26(1)

Art 26(6)

Art 26(7)

EU-Canada Comprehensive Economic and Trade Agreement (CETA) 2014

Art 8.20(3)

European Convention on Human Rights and Fundamental Freedoms 1950

Art 6(3)(c).

Article 45.1

4.749

European Convention on Offences Relating to Cultural Property 1985

Art 8(3)

Framework Agreement of the Pacific Alliance 2014

Additional Protocol

Art 10.25(5)

France-Argentina Bilateral Investment Treaty 1991

France-Mexico Bilateral Investment Treaty 1998

Art 2(1)

Art 3

Free Trade Area of the Americas Third Draft Agreement 2003 (FTAA)

Ch XVII, Art 5

Germany-Ghana Bilateral Investment Treaty 1967 $.2 .42,2.49,2.62$

Germany-Lebanon Bilateral Investment Treaty 1999

Art 9(3)

Germany-Philippines Bilateral Investment Treaty 1997 $2.42,2.45,2.49$

Art 1(1)

Germany-Ukraine Bilateral Investment Treaty 1993

$2.46,2.53,2.57$

Germany-Yugoslavia Bilateral Investment Treaty 1989

Greece-Albania Bilateral Investment Treaty 1995

Art 9(9)

Greece-Romania Bilateral Investment Treaty 1997

Art 9(1) $4.444-4.447,25.125$

Art 9(2)

Hague Convention on Conflict of Laws 1930

Art 1

Hague Convention on Peace 1899

Hague Convention on Peace 1907

Hong Kong-Chile Bilateral Investment Treaty 2016

Art 25(1)

Art 25(2)

Art 25(3)

Israel-Uzbekistan Bilateral Investment Treaty 1994

Art 8(1) 
Israel-Uzbekistan Bilateral Investment Treaty 1997

Art 1(1)

Art 8(1)

Italy-Chad Bilateral Investment Treaty 1969.

Italy-Cuba Bilateral Investment Treaty 1993.

.2 .329

Italy-Jordan Bilateral Investment Treaty 1996 .2 .93

Italy-Morocco Bilateral Investment Treaty 1990 .2 .45

Italy-Romania Bilateral Investment Treaty 1990

Art 8

Art 8(1) 4.451

Art 8(2) 4.451

Art 8(3)

Italy-Turkey Bilateral Investment Treaty 1995

Art 8.4

Japan-Oman Bilateral Investment Treaty 2015 .....

Kazakhstan-Uzbekistan Bilateral Investment Treaty 1997

Art 1(5)

Kenya-UK Bilateral Investment Treaty 1999

Korea-Colombia Free Trade Agreement 2013

League of Nations Covenant

Art 18

Mauritius-Egypt Bilateral Investment Treaty 2014

Art 10

Mexico-Germany Bilateral Investment Treaty 1998

Art 1

Mexico-Greece Bilateral Investment Treaty 2000

Art 13(2)

Mexico-United Arab Emirates Bilateral Investment Treaty 2016

Art 14(2)

Morocco-Nigeria Bilateral Investment Treaty 2016

Art 27(2)(a)

Art 27(2)(b)

Netherlands-Slovakia Bilateral Investment Treaty 1991

Art 8

Netherlands-Slovakia Bilateral Investment Treaty [date]

Art 8(1)

Netherlands-Turkey Bilateral Investment Treaty 1986

Art 8(3)

Netherlands-Venezuela Bilateral Investment Treaty 1991

New York Convention on Recognition and Enforcement of Foreign Arbitral Awards 1958

4.1352, 4.1372, 7.13, 7.19, 7.23, 7.29, 9.11, 16.11, 16.13

Art V...... $4.1336-4.1337,4.1354$

Art $\mathrm{V}(1)(\mathrm{b})$ $4.969,4.1035$

Art $V(1)(c)$

Art $V(1)(d)$

Art V(1)(e)

Art $V(2)(b)$ 
Nigeria-Morocco Bilateral Investment Treaty 2016

Art 27(2)(d)

Nigeria-Singapore Bilateral Investment Treaty 2016

Art 14(1) . .4 .84$

Art 14(2)

Art 16(4)

Art 18(1)

Art 22(8)

North American Free Trade Agreement 1994 (NAFTA)

Art 1103(2)

$2.77,2.301,2.320,2.323$

Art 1116

.2 .104

Art 1117

2.186

Art 1121

2.186

Art 1121(2)(b)

$2.300,4.118$

Art 1124

.2 .300

Art 1124(1)

4.106

Art 1126

1.115

Art 1130

4.118-4.119, 4.121-4.122

Art 1131

$7.19,7.29$

Art 1136(3)

4.256

Art 1137(4)

4.1318-4.1319, 4.1362

OECD Convention on Combating Bribery of Foreign Public Officials in International

Business Transactions 1997.

Pakistan-Switzerland Bilateral Investment Treaty 1995

Art 11

Panama-Germany Bilateral Investment Treaty 1993

Art 1(4)(a).

Paris Convention on Nuclear Energy 1960

Art 15(b)

Philippines-Switzerland Bilateral Investment Treaty 1997

Art II

Philippines-Switzerland Bilateral Investment Treaty [date]

Art VIII

Romania-Sri Lanka Bilateral Investment Treaty 1981

Art 7(2)

Romania-Turkey Bilateral Investment Treaty 1991

Russia-Mongolia Bilateral Investment Treaty 1995

Russia-Mongolia Bilateral Investment Treaty [date]

Art 6

Russia-Ukraine Bilateral Investment Treaty 1998

Slovakia-Czech Republic Bilateral Investment Treaty 1992

Art 8(2)

Art 8(3)

Slovakia-Greece Bilateral Investment Treaty 1991

Slovakia-Iran Bilateral Investment Treaty 2016

Art 18(1)

Art 18(2) 
Art 18(3) 4.84

Art 18(4) 4.84

Art 18(5)

Sri Lanka - UK Bilateral Investment Treaty 1980

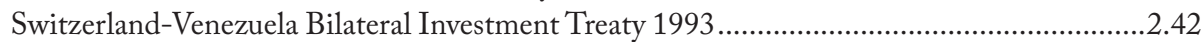

Tanzania-UK Bilateral Investment Treaty 1994.

Treaty on the Functioning of the European Union 2007

Art 3(2)

Art 207

Art 267

Art 344

Turkey-Australia Bilateral Investment Treaty 2005

Art 1

Art 2(1)

Turkey-Azerbaijan Bilateral Investment Treaty 2011

Art 1

Turkey-Jordan Bilateral Investment Treaty 1993

Art 1(2)

Turkey-Turkmenistan Bilateral Investment Treaty 2015

$\operatorname{Art} \operatorname{VII}(2)$

Turkey-US Bilateral Investment Treaty 1985

UK-Argentina Bilateral Investment Treaty 1990

Art 3(2)

UK-India Bilateral Investment Treaty 1994

Art 1(b)

Art 13

UK-Indonesia Bilateral Investment Treaty 1976

Art 2(1)

UK Model Bilateral Investment Treaty

Art 3(3)

Ukraine-Denmark Bilateral Investment Treaty 1992

Art 1(1)

United Arab Emirates-Japan Bilateral Investment Treaty 2016

Art 13(1)

$\operatorname{Art} 13(2)$

United Nations Convention on Transparency in Treaty-based Investor-State Arbitration 2014 ..

$4.569,4.778,4.790-4.793,24.105-24.106,26.89$

Art 2(1)

Art 2(2)

Art 2(3)(1)(a)-(b)

Art 3.1

.4 .790

Art 3.2

4.790

US-Argentina Bilateral Investment Treaty 1991

Art VII $(8)$

$\operatorname{VII}(3)(a)$

$\operatorname{VII}(3)(\mathrm{b})$

US Bilateral Investment Treaty 2004 
US-Ecuador Bilateral Investment Treaty 1993 $2.314-2.315,8.20$

US Model Bilateral Investment Treaty $2.32,2.38,2.304,24.98$

Art 1

Art 2

Art 26(1)

.2 .95

Art 27(1) 4.84

Art 27(2)

4.84

Art 27(3)

$4.84-4.85$

Art 28(2)

.26 .89

Art 29(1)

26.89

Art 33

26.89

US-Oman Free Trade Agreement 2006

Art 1.3

Art 10.1

US-Romania 1992

Art VI(2).

Art VI(3)

US-Uruguay Bilateral Investment Treaty 2005

Art 1

2.32

Uzbekistan-Kazakhstan Bilateral Investment Treaty 1997

$2.42,2.49-2.50$

Vienna Convention on the Law of Treaties 1969........ 0.14, 0.77, 2.83, 2.166, 10.09, 10.11, 10.27 Art 4 $10.33,10.85$

Art 7 .22 .74

Art 27 4.250

Art 28

2.91

Art 31

$.0 .13,0.69,4.03$

Art 31(1) 4.1223

Art 32 4.1223

Art 33 $0.08,4.1350$

Art 33(1)

10.102

Art 33(4)

10.104

Art 39

$2.247,9.07$

Art 40(2)

9.04

Art 41

4.1316

Art 54

$2.247,10.34$

Art 54(a) 10.33

Art 59 10.34

Art 70 10.34

Art 70(2) 10.34

Art 71(a) 10.34

Art 76 .10 .84

Art 76(2) 10.85

Art 77 10.97

Art 77(1)(e) 10.96-10.97

Art 77(1)(f) 10.97

Art 81 .10 .05 
Julien Fouret, Rémy Gerbay, and Gloria M. Alvarez - 9781786435248 Downloaded from PubFactory at $04 / 26 / 2023$ 12:56:22PM 